\title{
BMJ Open Associations between anthropometric indices, blood pressure and physical fitness performance in young Swiss men: a cross-sectional study
}

\author{
Kaspar Staub, ${ }^{1}$ Joël Floris, ${ }^{1,2}$ Nikola Koepke, ${ }^{1}$ Adrian Trapp, ${ }^{3}$ Andreas Nacht, ${ }^{4}$ \\ Susanna Schärli Maurer, ${ }^{5}$ Frank J Rühli, ${ }^{1}$ Nicole Bender ${ }^{1}$
}

To cite: Staub K, Floris J, Koepke N, et al. Associations between anthropometric indices, blood pressure and physical fitness performance in young Swiss men: a crosssectional study. BMJ Open 2018;8:e018664. doi:10.1136/ bmjopen-2017-018664

- Prepublication history and additional material for this paper are available online. To view these files, please visit the journal online (http://dx.doi. org/10.1136/bmjopen-2017018664).

Received 14 July 2017 Revised 24 April 2018 Accepted 25 April 2018

Check for updates

${ }^{1}$ Institute of Evolutionary Medicine, University of Zurich, Zürich, Switzerland

${ }^{2}$ Department of Economics, University of Zurich, Zürich, Switzerland

${ }^{3}$ Swiss Armed Forces, Recruiting Centre 2 Sumiswald and Army Medical Services, Sumiswald,

Switzerland

${ }^{4}$ Swiss Armed Forces, Recruiting Centre 6 Mels and Army Medical Services, Mels, Switzerland

${ }^{5}$ Swiss Armed Forces, Recruiting Centre 4 Windisch and Army Medical Services, Windisch,

Switzerland

Correspondence to

Dr Nicole Bender;

nicole.bender@iem.uzh.ch

\section{ABSTRACT}

Objectives To assess the benefit of waist circumference (WC) measurements during routine conscription medical examination in two military conscription centres in Switzerland. We compared the prevalence of overweight and obesity assessed by body mass index (BMI) with the prevalence of elevated disease risks assessed by WC and waist-to-height ratio (WHtR). We investigated how these measures were associated with systolic blood pressure, physical fitness performance and socioeconomic determinants.

Design Cross-sectional survey.

Setting Two Swiss conscription centres in 2016.

Participants 1548 Swiss male conscripts, 18-22 years old.

Main outcome Prevalences of elevated WC, WHtR and $\mathrm{BMI}$ values according to WHO categories. Secondary outcomes include systolic blood pressure, physical fitness performance and endurance performance.

Results Using BMI cut-points, $25.0 \%$ of all conscripts were overweight or obese. When applying WC cut-points, $9.2 \%$ had an increased disease risk, while $14.8 \%$ of the conscripts were at risk using WHtR cut-points. In the BMI range of $25.0-27.4 \mathrm{~kg} / \mathrm{m}^{2}, 3.6 \%$ showed an increased disease risk when using WC and $24.6 \%$ when using WHtR cut-points. Of the conscripts with a BMl of $27.5-29.9 \mathrm{~kg} /$ $\mathrm{m}^{2}, 72.4 \%$ had an increased disease risk using WHtR, and $42.5 \%$ when using WC cut-points. Determinants of elevated BMI, WC and WHtR were low occupational status, rural residential area, older age and location in central and Northwest Switzerland. Systolic blood pressure increased with increasing BMI, WC and WHtR. Physical fitness and endurance test performances decreased with increasing BMI, WC and WHtR.

Conclusion In addition to BMI, WC and WHtR add relevant information to the health assessment of young men. However, the prevalence of overweight/increased health risk differed when using BMI, WC or WHtR. Further studies should include measures of body composition to test whether these differences arise from muscular young men within the overweight BMI range, who had a normal WC.

\section{INTRODUCTION}

Obesity and its detrimental health effects such as cardiovascular diseases (CVD) and
Strengths and limitations of this study

- This is the first assessment of waist circumference (WC) measurements in Swiss-German male conscripts, including over 1500 individuals.

- We compared WC and waist-to-height ratio with body mass index to assess the association with systolic blood pressure and physical fitness test performance.

- We assessed several occupational and regional determinants of weight parameters in young Swiss men.

- We cannot exclude WC measurement errors even though we instructed the measuring staff, created an instructive poster and visited measurement sites in the first weeks to ensure a correct measurement technique.

- We had only a limited number of potential confounding variables to include in our analyses.

type II diabetes are increasing worldwide ${ }^{1}$ and in Switzerland. ${ }^{2}$ Data from 2007 show that in Switzerland 27000 cases of type II diabetes, 63000 cases of hypertension and 37000 cases of dyslipidaemia would have been avoided if overweight and obesity could have been kept at the levels reported in $1992 .{ }^{3}$ In 2006, direct and indirect costs caused by overweight and obesity reached 5.8 billion Swiss francs, ${ }^{4}$ and in 2012 these costs increased to 8.0 billion. $^{5}$ Recent studies of schoolchildren ${ }^{67}$ and Swiss Armed Forces conscripts ${ }^{89}$ have shown that rates of overweight and obese children and young adult men have not increased during the past 5 years. Still, in 2015 every fourth conscript was categorised as overweight or obese in Switzerland, therefore potentially carrying an increased risk of disease. ${ }^{10}$

Epidemiological studies usually assess the prevalence of overweight and obesity by calculating the body mass index (BMI, body weight divided by the square of the body height). However, BMI is only a rough 
measure of overweight. It cannot distinguish between fat mass and muscle mass, and therefore does not allow drawing health-relevant conclusions about body composition. ${ }^{11}$ Waist circumference (WC) and waist-to-height ratio $(\mathrm{WHtR})$ are increasingly used to make better inferences about health-related body shape and fat distributions, especially regarding abdominal fat. Although WC is an absolute measure, WHtR is corrected for body height.

BMI and WC differ regarding disease risk assessment within a population. ${ }^{12}$ Having more or less muscle mass is one of the possible explanations ${ }^{13}$ because physical activity also correlates more strongly with WHtR than BMI. ${ }^{14}$ In fact, abdominal fat is more strongly associated with morbidity and mortality than BMI. ${ }^{15} 16$ However, to date it remains unclear which anthropometric measurements best indicate risks for diseases such as CVD or type II diabetes. ${ }^{1718}$ Although some studies find a stronger association with CVD risk factors in WHtR than BMI, others find similar associations. ${ }^{19-24}$ Evidence from reviews show that the levels of association with morbidity and mortality outcomes even differ based on the disease of interest. ${ }^{25} 26$

Most existing studies of overweight and obesity in Switzerland rely on partially self-reported BMI, and the number of existing studies of WC per se and its difference from BMI among the Swiss population and particularly among young people is still small. ${ }^{27-33}$ This is striking since obese young people tend to carry their overweight into adulthood, which increases risk for morbidity and mortality in later life. ${ }^{34} 35$ Therefore, continuous and precise monitoring of overweight and obesity among young people as a basis for focused public health interventions could have an impact on the health status of the whole population over the coming decades.

In summer 2016, we investigated the feasibility and the benefit of WC measurements during the routine conscription medical examination in two Swiss Armed Forces conscription centres. Our specific research aims were to compare the prevalence of overweight and obesity based on BMI with the prevalence of elevated disease risks based on WC and WHtR to assess which of these measures better correlates with systolic blood pressure and physical fitness test performance in young men. We also investigated how these measures are associated with other variables such as age, and occupational and regional determinants.

\section{MATERIALS AND METHODS}

All Swiss men reaching the age of 19 in a given year are summoned for conscription in one of six Swiss Armed Forces conscription centres based on their official place of residence. Earlier or later conscription is possible, and total coverage of a given birth year is up to $95 \% .^{8}$ The conscription process in Switzerland lasts 2-3 days, depending on the centre. During the medical examination, standard protocols and measurement tools are used in all centres. In addition to health data, occupation and place of residence are recorded. Body height and weight are measured using a stadiometer and a regularly calibrated scale (both SECA), in underwear and without shoes. Among other tests, a physical fitness test consisting of several parts is carried out (more details follow). Blood pressure is measured with calibrated, automatic, upper-arm blood pressure monitors. If the blood pressure is above either the limit of $140 / 90$, the measurement is repeated up to two times, and all results are recorded.

After the Swiss Armed Forces approved our study protocol, our study was conducted at the Swiss conscription centres of Mels (GR) and Windisch (AG) from July 2016 to August 2016. The WC measurements for our study were performed by trained army medical personnel, or by soldiers serving in the conscription process who are specifically trained to measure WC accurately. All army conscripts assessed in the two conscription centres during the study period were included in our study. We regularly observed the measuring procedure during the first weeks to ensure correct measurement technique. We instructed army personnel to use WHO standard protocol to allow an international comparison of our results with other studies. ${ }^{36}$ In WHO guidelines, WC is measured at the midpoint between the lower end of the rib cage and the upper end of the pelvis, assessed at the lateral side of the body. To ensure a uniform application of this protocol, we created an instructive poster that was displayed in the measuring rooms of both centres. We supplied the army with SECA 201 measuring tapes that allow an automatic and standardised tightening of the tape. The newly collected WC measurements were linked to the routinely collected health and occupational data by the army (Logistikbasis der Armee, LBA San), anonymised, and then sent to the investigator team at the Institute of Evolutionary Medicine at the University of Zurich for analysis.

We calculated BMI $\left(\mathrm{kg} / \mathrm{m}^{2}\right)$ from body height and weight and categorised BMI in accordance with WHO guidelines. ${ }^{1}$ Because we were particularly interested in the overweight BMI range from 25.0 to $29.9 \mathrm{~kg} / \mathrm{m}^{2}$ (in which muscular young men might be misclassified by BMI), we subdivided the overweight WHO BMI category in two subgroups, overweight I $\left(25.0-27.4 \mathrm{~kg} / \mathrm{m}^{2}\right)$ and overweight II $\left(27.5-29.9 \mathrm{~kg} / \mathrm{m}^{2}\right)$ for our analyses. From WC and body height, we calculated WHtR $(\mathrm{cm} / \mathrm{cm})$ and categorised WC $(\mathrm{cm})$ according to WHO guidelines and WHtR according to Ashwell et al $l^{17}$ (table 1). The physical fitness test at conscription (Test Fitness Rekrutierung, TFR) assesses the physical performance of Swiss conscripts in five aspects of fitness: speed and strength of legs and arms, global trunk strength, coordination and endurance. Performance in each is evaluated with the help of a point scale (0-25 per aspect; in total, maximum 125). This test was previously validated. ${ }^{37}$ We analysed the TFR total and the endurance test separately. The army ranks the TFR by five performance categories ranging from insufficient to excellent (table 1). If more than one measurement of systolic blood pressure was taken from one conscript, the intraindividual mean was calculated and used in the analysis. Systolic blood pressure was categorised into normal $(<140 \mathrm{~mm} \mathrm{Hg})$ and elevated $(\geq 140 \mathrm{~mm} \mathrm{Hg})$ values. ${ }^{38}$ 


\begin{tabular}{|c|c|}
\hline BMI $\left(\mathrm{kg} / \mathrm{m}^{2}\right)$ & Definition/disease risk \\
\hline$<18.5$ & Underweight \\
\hline $18.5-24.9$ & Normal weight, reference group \\
\hline \multirow[t]{3}{*}{ 25.0-29.9 } & Overweight \\
\hline & (Overweight I, 25.0-27.4) \\
\hline & (Overweight II, 27.5-29.9) \\
\hline$\geq 30.0$ & Obesity \\
\hline \multicolumn{2}{|l|}{ WC (cm) } \\
\hline$<94.0$ & Risk not increased, reference group \\
\hline $94.0-101.9$ & Risk increased \\
\hline$\geq 102.0$ & Risk strongly increased \\
\hline \multicolumn{2}{|l|}{ WHtR (cm/cm) } \\
\hline$<0.50$ & Risk not increased, reference group \\
\hline $0.50-0.59$ & Risk increased \\
\hline$\geq 0.60$ & Risk strongly increased \\
\hline Total points TFR & Category \\
\hline $0-34$ & Insufficient \\
\hline $35-64$ & Sufficient \\
\hline $65-79$ & Good \\
\hline 80-99 & Very good \\
\hline $100-125$ & Excellent \\
\hline
\end{tabular}

Body mass index (BMI) and waist circumference (WC) according to $\mathrm{WHO},{ }^{1}$ and waist-to-height ratio $(\mathrm{WHtR})$ according to Ashwell et al. ${ }^{17}$

For all analyses, we subdivided the overweight WHO BMI category (BMI $25.0-29.9 \mathrm{~kg} / \mathrm{m}^{2}$ ) into two subgroups (BMI $25.0-27.4 \mathrm{~kg} / \mathrm{m}^{2}$ and $27.5-29.9 \mathrm{~kg} / \mathrm{m}^{2}$ ). Lower part: official categorisation of the total points of the conscription fitness test (Test Fitness Rekrutierung, TFR).

Occupational status was based on the self-reported occupation of the conscripts. We used the SocioEconomic Index of Occupational Status to categorise each occupation. ${ }^{39}{ }^{40}$ We also divided this index into tertiles. Students and conscripts without a profession or with an imprecise indication of profession (in terms of the official Swiss classification) were categorised in separate groups. We defined cases in which the entry for occupation was labelled 'insufficient' $(n=23)$ or 'without' $(n=102)$ as imprecise. ${ }^{8}$ The postal codes of the place of residence were categorised according to the Swiss Federal Office of Statistics to define the location of residence by major region (Grossregion) and urbanicity of the residence. ${ }^{10}$

\section{Statistical analysis}

We calculated descriptive statistics (means, SD, medians) for BMI, WC and WHtR, and compared the prevalence of elevated body weight/disease risk against each other in cross tabulations. We also calculated Pearson correlations between BMI and WC, and between BMI and WHtR. We calculated kappa coefficients ${ }^{41}$ to assess the agreement between classifications into normal weight, overweight/ increased disease risk and obesity/strongly increased disease risk. The kappa coefficient was classified according to Altman's reference: $0.41-0.60=$ moderate agreement, $0.61-0.80=$ good agreement, $>0.80=$ excellent agreement. The associations among BMI, WC and WHtR, and determinants such as occupational status, urbanicity, major region and age were assessed by linear regression. The same method was used when analysing the association between the three anthropometric measures and systolic blood pressure and physical fitness test performance as outcomes. For this outcome analysis, we combined the underweight BMI category $\left(\mathrm{BMI}<18.5 \mathrm{~kg} / \mathrm{m}^{2}\right)$ and the normal BMI category $\left(18.5-24.9 \mathrm{~kg} / \mathrm{m}^{2}\right)$ into one 'normal' BMI category of BMI $<25.0 \mathrm{~kg} / \mathrm{m}^{2}$ to have reference groups comparable to WC $(<94 \mathrm{~cm})$ and $\mathrm{WHtR}$ $(<0.50)$. The linear regression that assessed blood pressure and physical fitness test results among overweight (BMI $25.0-29.9 \mathrm{~kg} / \mathrm{m}^{2}$ ) young men above or below the respective $\mathrm{WC}$ and $\mathrm{WH} \mathrm{HR}$ thresholds of $94.0 \mathrm{~cm}$ and 0.50 against normal BMI conscripts was additionally adjusted for age, occupational status and region of residence. We used the STATA (V.14.2) statistical package for all statistical analyses.

\section{Patient and public involvement}

According to Swiss federal law (Bundesgesetz über die militärischen Informationssysteme, BG 510.91, Art. 2, 9, 24-29), the Swiss Armed Forces are authorised to provide the anonymised data for academic research. The Swiss Armed Forces fully anonymised the records by removing all names, social security numbers, ID numbers and exact residential addresses. Because Swiss conscription is mandatory and the anthropometric measurements used in this study are non-clinical, governmental data, informed consent and ethical approval were not needed (Swiss data privacy act, SR 235.1; 19.6.1992 and Federal Act on Research involving Human Beings (Human Research Act, HRA) $810.30 ; 1.1 .2014) .{ }^{84243}$

\section{RESULTS}

In total, 1548 young men were conscripted in the two centres between July 2016 and August 2016 (606 at Mels and 942 at Windisch). WC was available for 1536 conscripts $(99.2 \%)$. The descriptive results of the anthropometric measurements and health parameters are reported in table 2 (upper table). The prevalence of overweight and obesity (BMI) and the prevalence of increased disease risk (WC and WHtR) differed (table 2, lower table). Using BMI cut-points, 25.0\% (95\% CI 22.8 to 27.1) of all conscripts were overweight or obese; using WC cut-points, $9.2 \%$ (95\% CI $7.8 \%$ to $10.7 \%$ ) had an increased disease risk; and using WHtR cut-points, $14.8 \%$ (95\% CI $13.0 \%$ to $16.7 \%$ ) had an increased disease risk. When comparing BMI and WC (figure 1 and table 3), a large percentage $(16.1 \%$, 95\% CI $14.5 \%$ to $17.9 \%$ ) of conscripts had a BMI above $25.0 \mathrm{~kg} / \mathrm{m}^{2}$ but no 
Table 2 Upper table: descriptive statistics for the anthropometric variables, systolic blood pressure and results of physical fitness test; lower table: absolute and relative frequencies for body mass index (BMI), waist circumference (WC) and waist-toheight ratio (WHtR) categories

\begin{tabular}{|c|c|c|c|c|c|c|}
\hline Continuous variables & $\mathbf{N}$ & Mean & SD & Minimum & Median & Maximum \\
\hline Height (cm) & 1548 & 178.9 & 6.6 & 157.0 & 179.0 & 203.0 \\
\hline Weight (kg) & 1548 & 75.0 & 13.5 & 44.0 & 73.0 & 150.0 \\
\hline $\mathrm{BMI}\left(\mathrm{kg} / \mathrm{m}^{2}\right)$ & 1548 & 23.4 & 3.9 & 15.8 & 22.6 & 45.8 \\
\hline WC $(\mathrm{cm})$ & 1536 & 80.9 & 9.7 & 60.8 & 79.0 & 139.7 \\
\hline WHtR $(\mathrm{cm} / \mathrm{cm})$ & 1536 & 0.5 & 0.1 & 0.3 & 0.4 & 0.8 \\
\hline Systolic blood pressure $(\mathrm{mm} \mathrm{Hg})$ & 1536 & 128.6 & 9.4 & 97.0 & 129.0 & 168.0 \\
\hline Physical fitness test TFR (0-125 Pts) & 1385 & 68.5 & 14.6 & 17.0 & 69.0 & 109.0 \\
\hline Endurance test (0-25 Pts) & 1386 & 14.0 & 4.4 & 2.0 & 14.0 & 25.0 \\
\hline Categorisation & BMI (n) & BMI (\%) & WC (n) & WC (\%) & WHtR (n) & WHtR (\%) \\
\hline Underweight & 63 & 4.1 & & & & \\
\hline Normal weight/no increased risk & 1099 & 71.0 & 1395 & 90.8 & 1309 & 85.2 \\
\hline Increased risk & 195 & 12.6 & 72 & 4.7 & 183 & 11.9 \\
\hline Overweight II & 88 & 5.7 & & & & \\
\hline Obesity/strongly increased risk & 103 & 6.7 & 69 & 4.5 & 44 & 2.9 \\
\hline Total & 1548 & 100.0 & 1536 & 100.0 & 1536 & 100.0 \\
\hline
\end{tabular}

Pts, points; TFR, Test Fitness Rekrutierung.

increased disease risk $(\mathrm{WC}<94.0 \mathrm{~cm})$, whereas only a few conscripts $(0.3 \%, 95 \%$ CI $0.1 \%$ to $0.7 \%)$ had a BMI under $25.0 \mathrm{~kg} / \mathrm{m}^{2}$ and an increased disease risk (WC $\left.\geq 94.0 \mathrm{~cm}\right)$. When comparing BMI with WHtR, $11.3 \%$ (95\% CI 9.6\% to $12.8 \%$ ) were overweight (BMI $\geq 25.0 \mathrm{~kg} / \mathrm{m}^{2}$ ) with no disease risk $(<0.50)$, whereas $1.0 \% \quad(95 \%$ CI $0.5 \%$ to $1.6 \%)$ were normal weight with an increased disease risk. Pearson correlation coefficients were high between BMI and WC $(r=0.908, p<0.001)$ and between BMI and WHtR $(\mathrm{r}=0.912, \mathrm{p}<0.001)$.

If we compare anthropometric measures in conscripts with excessive weight (BMI $\geq 25.0 \mathrm{~kg} / \mathrm{m}^{2}, \mathrm{n}=386$ ), in the overweight I category (BMI $25.0-27.4 \mathrm{~kg} / \mathrm{m}^{2}, \mathrm{n}=195$ ), only $3.6 \%$ had a WC of more than $94 \mathrm{~cm}$, and $24.6 \%$ had a WHtR over 0.5 (online supplementary figure S1). In the overweight II category (BMI $27.5-29.9 \mathrm{~kg} / \mathrm{m}^{2}$, $\mathrm{n}=87$ ), $72.4 \%$ of the conscripts had an increased disease risk (WHtR). In the obese BMI category (BMI $\geq 30.0 \mathrm{~kg}$ / $\left.\mathrm{m}^{2}, \mathrm{n}=102\right)$, only a minority ( $8.8 \%$ for $\mathrm{WC}$ and $1.0 \%$ for $\mathrm{WHtR}$ ) did not have an increased disease risk. The kappa coefficient for agreement between BMI and WC classifications was 0.40 overall (moderate agreement), but only 0.12 (poor) if restricted to conscripts with BMI $\geq 25.0 \mathrm{~kg}$ / $\mathrm{m}^{2} \quad(\mathrm{n}=384)$. Similarly, the kappa coefficient for agreement between classification according to BMI and to WHtR was 0.53 overall (moderate agreement), but only 0.08 (poor) if restricted to conscripts with BMI $\geq 25.0 \mathrm{~kg} /$ $\mathrm{m}^{2}(\mathrm{n}=386)$.

Linear regressions indicated only a weak association of occupational status with mean BMI (figure 2, left). In fact, only students showed a significant lower BMI than participants of medium occupational status. Conscripts living in urban areas showed a lower BMI than conscripts living in rural areas, and older conscripts showed a higher $\mathrm{BMI}$ than younger ones. The results are similar for WC and WHtR (figure 2, middle and right). Values of BMI, WC and WHtR were slightly higher in central Switzerland and the Northwest compared with other regions. However, most associations were not statistically significant or of moderate effect size. For detailed results, see online supplementary table S1.

Systolic blood pressure increased with increasing categories of BMI, WC and WHtR in linear regressions (figure 3, left), with significant differences between all weight/risk categories $(\mathrm{p}<0.001)$. Physical fitness test performance and endurance performance decreased with increasing categories of BMI, WC and WHtR (figure 3, middle and right). In general, coefficients in the BMI category overweight II $\left(27.5-29.9 \mathrm{~kg} / \mathrm{m}^{2}\right)$ were closer to those in the increased risk categories of $\mathrm{WC}$ and $\mathrm{WHtR}$, whereas the coefficients in the overweight I $\left(25.0-27.4 \mathrm{~kg} / \mathrm{m}^{2}\right)$ category were closer to the normal reference group. In the categories of obesity (BMI $\geq 30.0 \mathrm{~kg} / \mathrm{m}^{2}$ ) and of strongly increased disease risks in WC and WHtR, comparable coefficients emerged for all three weight parameters. For detailed results, see online supplementary table S2.

When the physical fitness test results were divided by categories of BMI (including overweight I and II), WC and WHtR (online supplementary figure S2), the previously described pattern was evident again, with $54.2 \%$ of all conscripts having good to excellent physical fitness results in the overweight I BMI category of $25.0-27.4 \mathrm{~kg} /$ $\mathrm{m}^{2}$, whereas $15.7 \%$ with a WC of $94.0-101.9 \mathrm{~cm}$ had good to excellent physical fitness results and $21.9 \%$ with a 
WC vs. BMI

Correlation: $\mathrm{R}=0.9078$

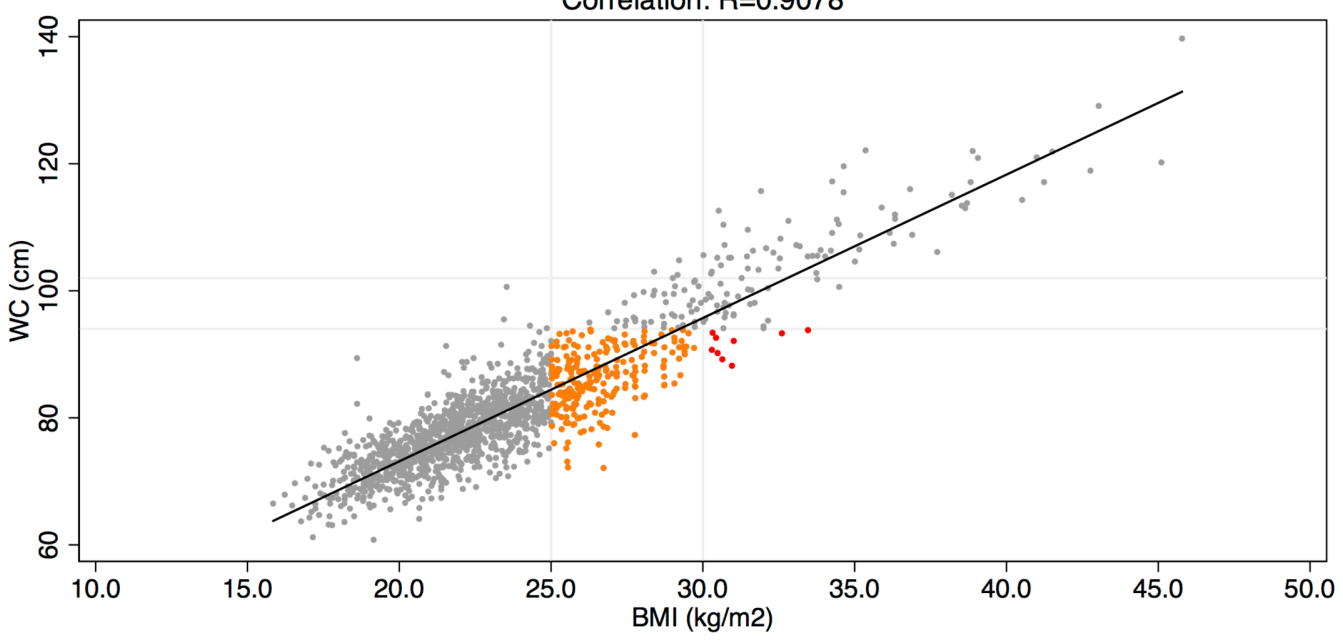

WHtR vs. BMI

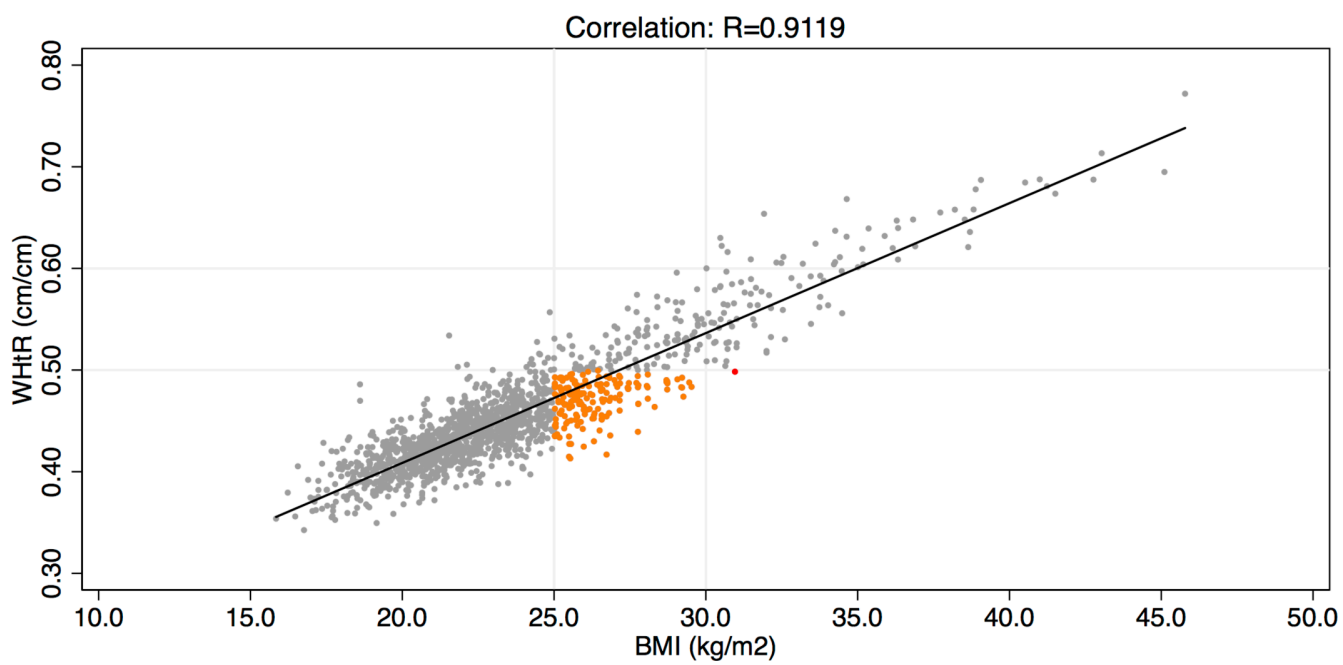

Figure 1 Scatter plots of body mass index (BMI) versus waist circumference (WC) (above) and BMI versus waist-to-height ratio (WHtR) (below). The BMI WHO thresholds for normal weight, overweight and obesity are displayed as reference lines on the $\mathrm{x}$-axes, the WC and WHtR threshold reference lines for increased disease risk and strongly increased disease risk are displayed on the $y$-axes. The solid black lines are the predicted linear regression lines. Orange and red data points indicate overweight and obese conscripts as measured by BMI, but with a WC/WHtR below the risk threshold.

WHtR of $0.50-0.59$ had good to excellent physical fitness results. A similar pattern is apparent for endurance test results, but not for systolic blood pressure.

When we subcategorised the conscripts in the overweight BMI category $25.0-29.9 \mathrm{~kg} / \mathrm{m}^{2}$ in overweight young men above or below the WC and WHtR thresholds of $94.0 \mathrm{~cm}$ and 0.50 , and ran linear regressions assessing blood pressure and physical fitness test results among these two subcategories against normal BMI conscripts $\left(\mathrm{BMI}<25.0 \mathrm{~kg} / \mathrm{m}^{2}\right)$ (figure 4), we found that 'overweight' conscripts with normal WC or WHtR clearly performed better in the overall physical fitness test and the endurance test than overweight conscripts with WC or WHtR in the increased risk category. A similar but less pronounced pattern became evident for blood pressure. The coefficients did not change substantially when adjusting the regressions for age, occupational status and region of residence.

\section{DISCUSSION}

Although in epidemiological settings, BMI is the most common weight and health risk indicator, ${ }^{44}{ }^{45}$ the assessments of WC and WHtR are recommended as additional measures to better capture the health risks of elevated visceral fat mass. ${ }^{1718}$ In our analysis, we found two thresholds for increased health risk according to WC or WHtR. Because WC and height are correlated, WHtR may be a more effective measurement, and it seems that WHtR best correlates with visceral fat percentage and total fat mass. ${ }^{46}$ Taking WHtR as the measure, $11.3 \%(\mathrm{n}=172)$ of all conscripts were classified as overweight by BMI without 
Table 3 Concordance of classification into weight/disease risk categories between body mass index (BMI) and waist circumference (WC) (upper table, $n=1536,100 \%$ ), and BMI and waist-to-height ratio (WHtR) (bottom table, $n=1536,100 \%$ ), respectively

\begin{tabular}{|c|c|c|c|c|c|c|}
\hline & BMI & & & & & \\
\hline WC & $<18.5$ & $18.5-24.9$ & $25.0-27.4$ & $27.5-29.9$ & $\geq 30.0$ & Total N (\%) \\
\hline$<94.0$ & $63(4.1)$ & 1085 (70.6) & $188(12.2)$ & $50(3.3)$ & $9(0.6)$ & 1395 \\
\hline $94.0-101.9$ & & $4(0.3)$ & $7(0.5)$ & $33(2.1)$ & $28(1.8)$ & 72 \\
\hline$\geq 102$ & & & $0(0.0)$ & $4(0.3)$ & $65(4.2)$ & 69 \\
\hline Total N(\%) & 63 & 1089 & 195 & 87 & 102 & $1536(100)$ \\
\hline WHtR & $<18.5$ & $18.5-24.9$ & $25.0-27.4$ & $27.5-29.9$ & $\geq 30.0$ & Total N (\%) \\
\hline$<0.50$ & $63(4.1)$ & 1074 (69.9) & 147 (9.6) & $24(1.6)$ & $1(0.1)$ & 1309 \\
\hline $0.50-0.59$ & & $15(1.0)$ & $48(3.1)$ & $63(4.1)$ & $57(3.7)$ & 183 \\
\hline$\geq 0.60$ & & & $0(0.0)$ & & $44(2.9)$ & 44 \\
\hline Total N (\%) & 63 & 1089 & 195 & 87 & 102 & $1536(100)$ \\
\hline
\end{tabular}

being at increased health risk, and $1.0 \%(\mathrm{n}=15)$ were classified as normal weight by BMI despite being at increased health risk. Overweight (BMI $25.0-29.9 \mathrm{~kg} / \mathrm{m}^{2}$ ) conscripts with a normal WC below $94 \mathrm{~cm}$ or a WHtR below 0.50 performed markedly better in the total physical fitness and endurance tests than overweight conscripts with elevated WC or WHtR values.

Only a few Swiss epidemiological data sets on WC measurements exist that can be compared with our study. Existing Swiss studies including WC data are based on schoolchildren, ${ }^{27} 2847$ older people, ${ }^{29}$ the Swiss Salt Survey, ${ }^{30} 33$ the Swiss CoLaus study ${ }^{31} 48$ and the Swiss MenuCH nutrition survey. ${ }^{32}$ Because young men are rarely included in epidemiological health research studies, large reference samples of WC in young men do not exist for Switzerland. If we compare the prevalence of increased disease risk according to WC between men aged 15-29years from the Swiss Salt Survey $(n=165)$ and men aged 18-34years from the MenuCH Survey with our subjects, the results are similar $(7.3 \%$ vs $8.9 \%$ vs $4.7 \%$ in the $94.0-101.9 \mathrm{~cm}$ category, $6.1 \%$ vs $3.9 \%$ vs $4.5 \%$ in the $\geq 102.0 \mathrm{~cm}$ category). A recently published study of Swiss conscripts in the Italian part of Switzerland had a sample size similar to our study and showed similar results. ${ }^{34}$ The mean value for WC in that study was $82.0 \mathrm{~cm}$, compared with $80.9 \mathrm{~cm}$ in our study. The proportion of Swiss Italian conscripts with $\mathrm{WC} \geq 102.0 \mathrm{~cm}$ was $3.6 \%$, compared with $4.5 \%$ in our study. Internationally, the study on Austrian 18-year-old conscripts that was performed between 2001 and 2005 is comparable to our study and showed similar results. ${ }^{50}$ The mean WC value in Austrian conscripts was $81.4 \mathrm{~cm}$, compared with $80.9 \mathrm{~cm}$ in our study. These figures show that our sample is comparable to other samples of the same sex and similar ages.

One problem with BMI is that it cannot distinguish between muscle mass and fat mass. Our study revealed that this problem might be of special relevance in young men with BMI of $25.0-27.4 \mathrm{~kg} / \mathrm{m}^{2}$. Our analysis indicated
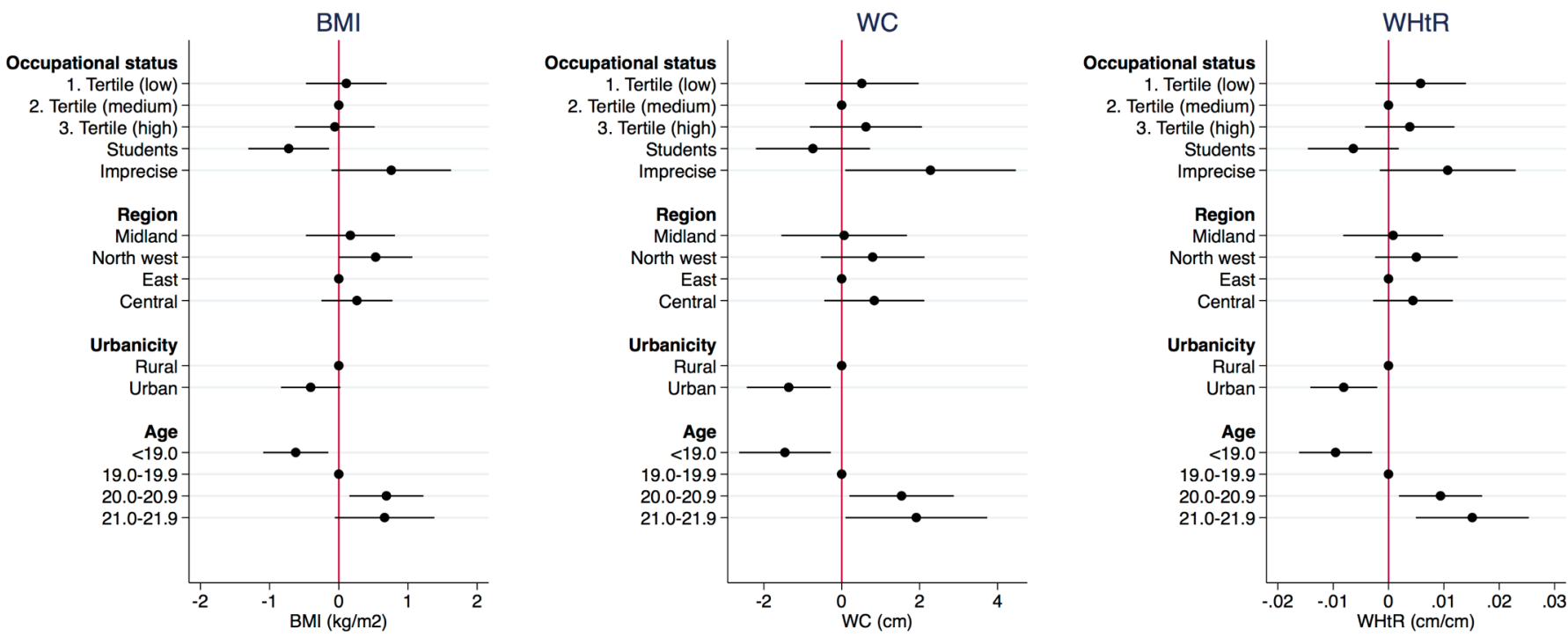

Figure 2 Coefficient plots showing the results of the linear regressions for the occupational and regional determinants of body mass index (BMI) (left), waist circumference (WC) (middle) and waist-to-height ratio (WHtR) (right). 

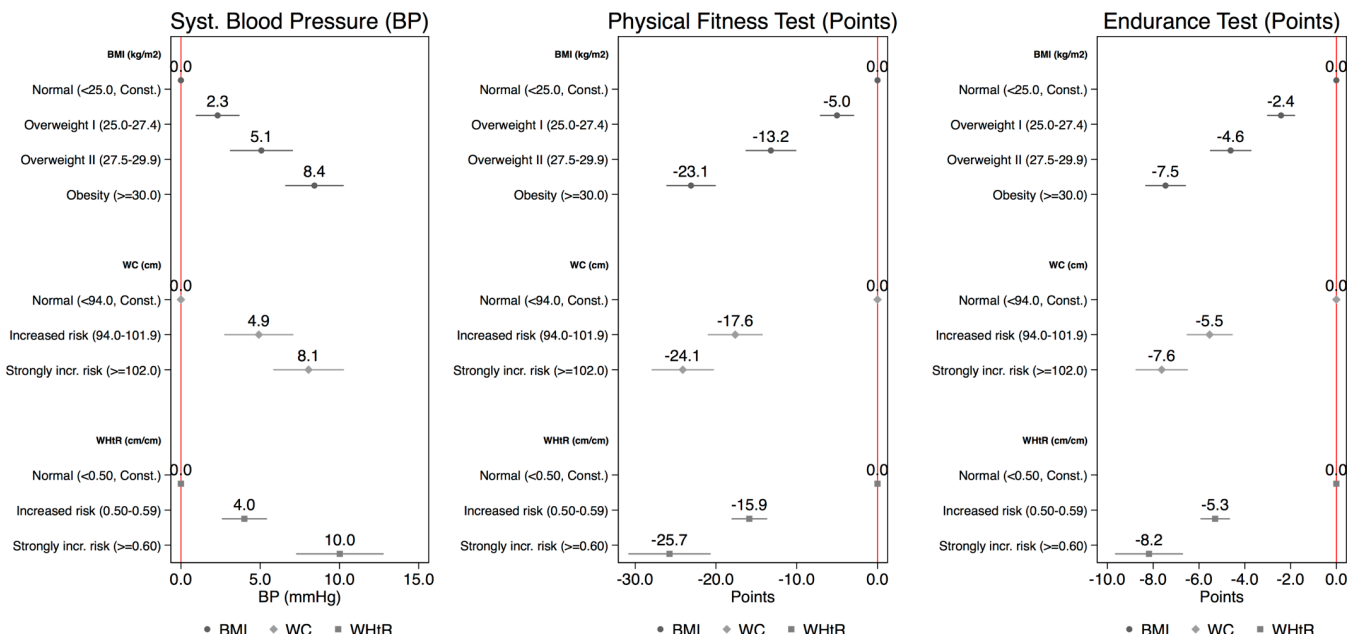

Figure 3 Coefficient plots showing the results of the linear regressions for blood pressure (left), physical fitness test (middle) and endurance test (right), according to body mass index (BMI) (above), waist circumference (WC) (middle) and waist-toheight ratio (WHtR) (below).

that both WC and WHtR classify conscripts as being at an increased disease risk only from BMI $27.5 \mathrm{~kg} / \mathrm{m}^{2}$ onwards. The associations of variables such as physical fitness test performance and endurance test performance with BMI, WC or WHtR differed more in the category of overweight/increased disease risk than in the category of obesity/strongly increased risk. Furthermore, the analysis showed that health risks in overweight conscripts might be underestimated if assessed by BMI alone because of the inclusion of the healthy muscular men in this category. WC and WHtR seem to classify health risks better because healthy muscular men are not included in the slightly elevated risk category. In the British National Diet and Nutrition Survey, $67 \%$ of men were overweight and $24 \%$ were obese, whereas $53 \%$ had a $\mathrm{WC} \geq 94$, and $30 \%$ had a WC $\geq 102$. According to the WHtR, $76 \%$ had a value $\geq 0.5 \%$ and $20 \%$ had a value $\geq 0.60 .{ }^{42}$ These figures are higher than our results because men of all age groups were included. However, the differences among obesity/ increased risks according to $\mathrm{BMI}, \mathrm{WC}$ and $\mathrm{WHtR}$ are not as strong as in our study, confirming that the problem of muscular men in the overweight category of BMI is
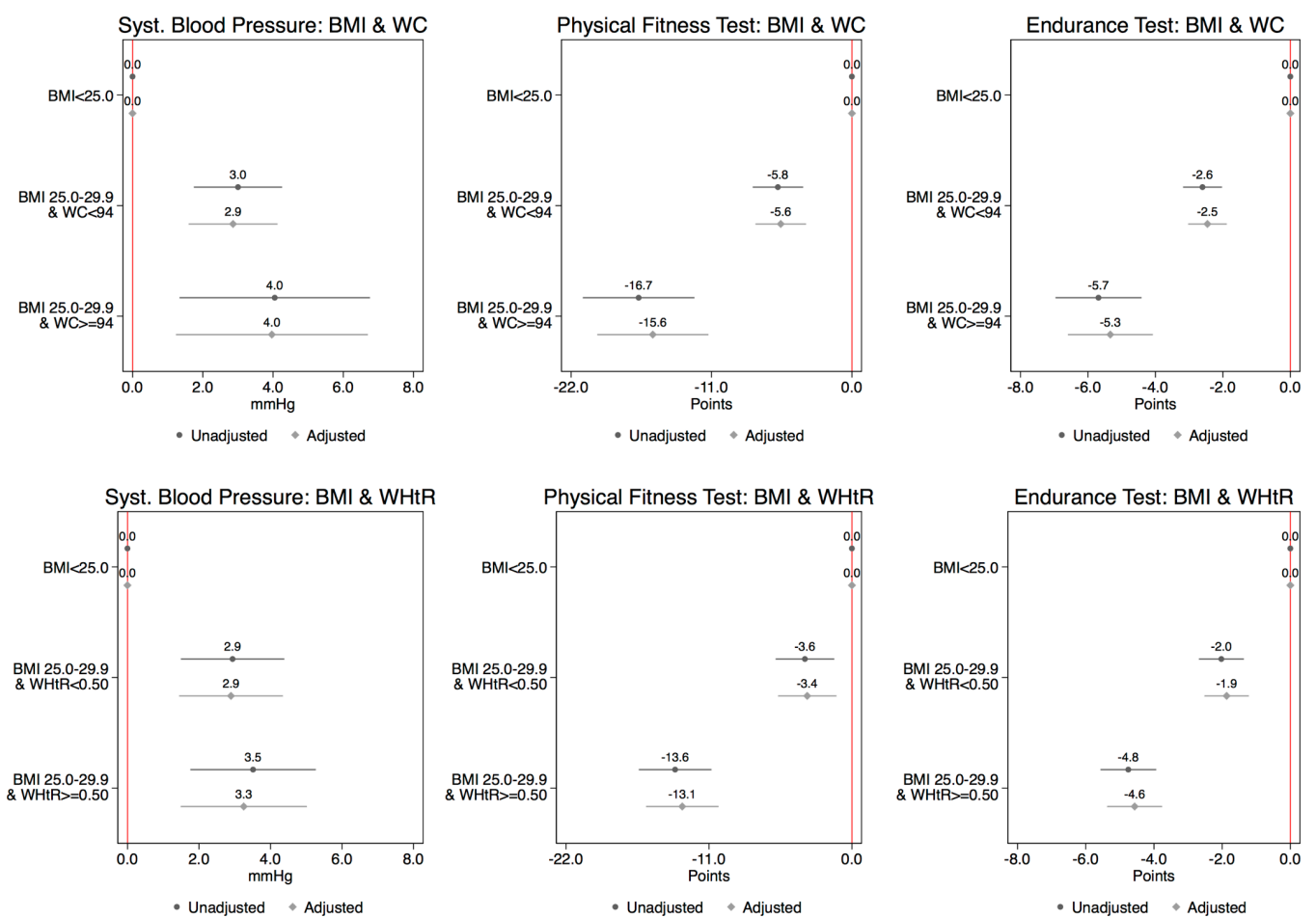

Figure 4 Coefficient plots showing the results of linear regressions comparing blood pressure and physical fitness test results among overweight (BMI $25.0-29.9 \mathrm{~kg} / \mathrm{m}^{2}$ ) young men above or below the $W C$ and $W H t R$ thresholds of $94.0 \mathrm{~cm}$ and 0.50 against normal BMI conscripts. In a second step, the regressions were additionally adjusted for age, occupational status and region of residence. BMI, body mass index; WC, waist circumference; WHtR, waist-to-height ratio. 
probably an issue in young men only. We conclude that in the BMI range of $25.0-27.4 \mathrm{~kg} / \mathrm{m}^{2}$, the truly overweight conscripts should be differentiated from the muscular conscripts to better assess whether and to what extent slight overweight increases health risks in both groups (taking into account additional information such as family history, smoking, nutrition, etc).

The strength of our study is that this is the first investigation of WC measurements in Swiss-German conscripts, totalling over 1500 individuals, which compared WC and WHtR with BMI to assess the association with systolic blood pressure and physical fitness test performance, as well as with occupational and regional determinants of weight parameters. One limitation was that we could not exclude interobserver errors in WC measurement even though we instructed the measuring staff, created an instructional poster and visited measurement sites in the initial weeks. In addition, because of a tight time schedule during the conscription process it was not possible to measure the WC of each conscript two times, by two persons. Generally, circumference measurements suffer from methodological challenges such as interobserver and intraobserver variability. ${ }^{52}$ It is therefore relevant to use standardised measurement protocols, well-trained measuring staff and validated measuring instruments. ${ }^{53}$ Another limitation was that we corrected for only a limited number of potentially confounding variables in our analyses. For instance, a number of conscripts were still undergoing professional training and it was not possible to classify these conscripts into occupational groups. We lacked further indicators such as income.

In addition to BMI, the measurement of $\mathrm{WC}$ and the calculation of WHtR adds relevant information to the health assessment in young men. Our results need to be confirmed at the national level. To verify our findings and find the best combination of weight and health indicators to predict health outcomes in epidemiological studies, we recommend measurements of body shape, body composition and metabolic parameters. A combination of BMI, $\mathrm{WC}$ and WHtR performs best in assessing disease risk. ${ }^{54}$ In addition, repeated measurements of the same persons over longer periods can reveal which indicators perform best at predicting disease risk in later life and disclose potentially changing risk profiles. Further studies should include women, and cohort studies should assess changes in body shape and composition over the life course from childhood to old age in the general population.

Acknowledgements Franz Frey, Franco Muggli, Tiziano Angelelli, Radoslaw Panczak, Marcel Zwahlen, Murielle Bochud, David Fäh, Isabelle Herter-Aeberli, Pedro Marquez-Vidal, Arnaud Chiolero, Jean-Pierre Pfammatter, Petra Cadetg, Matthias Bopp, Nadine Stoffel-Kurt, Andrea Poffet, Kathrin Favero, Thomas Wyss and Hans-Peter Stamm for technical input and discussions; Christopher Ritter for English editing; Andreas Stettbacher (Chief Medical Surgeon of the Swiss Armed Forces) for providing the data; MicroGIS SA for support in data management; and, in particular, the medical-technical staff of the conscript centres of Mels and Windisch for WC measurements.

Contributors Conceived and designed the WC measurements: KS, NB, JF and NK. Coordinated collection of data and prepared data for analysis: AN, SSM and AT. Analysed the data: KS and NB. Wrote first draft: NB and KS. Commented on paper: all authors. Obtained funding: KS, FJR and NB. Obtained routine army data: KS, FJR and NB.

Funding This work was supported by the Swiss Federal Office of Public Health FPO (contract number 16.008898) and Mäxi Foundation (Zurich).

Competing interests None declared.

Patient consent Not required.

Provenance and peer review Not commissioned; externally peer reviewed.

Data sharing statement The data are generated and owned by the Swiss Armed Forces. The data and the permission to use them are available from the Swiss Armed Forces (Logistikbasis der Armee-LBA San) on submission and approval of a study protocol.

Open access This is an Open Access article distributed in accordance with the Creative Commons Attribution Non Commercial (CC BY-NC 4.0) license, which permits others to distribute, remix, adapt, build upon this work non-commercially, and license their derivative works on different terms, provided the original work is properly cited and the use is non-commercial. See: http://creativecommons.org/ licenses/by-nc/4.0/

(c) Article author(s) (or their employer(s) unless otherwise stated in the text of the article) 2018. All rights reserved. No commercial use is permitted unless otherwise expressly granted.

\section{REFERENCES}

1. WHO. Obesity and overweight Fact sheet. Geneva: WHO, 2017.

2. Faeh DM A. Ernährung und Gesundheit. FOoP H, ed. Sechster Schweizerischer Ernährungsbericht. Bern: Merkur Druck, 2012:128-208.

3. Davin C, Vollenweider P, Waeber G, et al. Cardiovascular risk factors attributable to obesity and overweight in Switzerland. Nutr Metab Cardiovasc Dis 2012;22:952-8.

4. Schmid A, Schneider H, Golay A, et al. Economic burden of obesity and its comorbidities in Switzerland. Soz Praventivmed 2005;50:87-94.

5. Schneider H, Venetz W. Cost of Obesity in Switzerland in 2012. Rheinfelden, 2014.

6. Aeberli I, Ammann RS, Knabenhans M, et al. Decrease in the prevalence of paediatric adiposity in Switzerland from 2002 to 2007. Public Health Nutr 2010;13:806-11.

7. Aeberli I, Henschen I, Molinari L, et al. Stabilization of the prevalence of childhood obesity in Switzerland. Swiss Med Wkly 2010;140:w13046.

8. Panczak R, Zwahlen M, Woitek U, et al. Socioeconomic, temporal and regional variation in body mass index among 188,537 Swiss male conscripts born between 1986 and 1992. PLoS One 2014;9:e96721.

9. Staub K, Bender N, Floris J, et al. From Undernutrition to Overnutrition: The Evolution of Overweight and Obesity among Young Men in Switzerland since the 19th Century. Obes Facts 2016;9:259-72.

10. Floris J, Koepke N, Bender N, et al. Der Body Mass Index der Schweizer Stellungspflichtigen 2015. Bericht zuhanden des Bundesamtes für Gesundheit. Bern: Bundesamt für Gesundheit, 2016.

11. Frankenfield DC, Rowe WA, Cooney RN, et al. Limits of body mass index to detect obesity and predict body composition. Nutrition 2001;17:26-30.

12. Kjaer IG, Kolle E, Hansen BH, et al. Obesity prevalence in Norwegian adults assessed by body mass index, waist circumference and fat mass percentage. Clin Obes 2015;5:211-8.

13. Pasco JA, Nicholson GC, Brennan SL, et al. Prevalence of obesity and the relationship between the body mass index and body fat: cross-sectional, population-based data. PLoS One 2012;7:e29580.

14. Lee O, Lee DC, Lee S, et al. Associations between Physical Activity and Obesity Defined by Waist-To-Height Ratio and Body Mass Index in the Korean Population. PLoS One 2016;11:e0158245.

15. Janssen I, Katzmarzyk PT, Ross R. Waist circumference and not body mass index explains obesity-related health risk. Am J Clin Nutr 2004;79:379-84.

16. Ulijaszek SJ, Henneberg M, Henry CJ. One reason why waist-toheight ratio is usually better related to chronic disease risk and outcome than body mass index. Int J Food Sci Nutr 2013;64:269-73.

17. Ashwell M, Gunn P, Gibson S. Waist-to-height ratio is a better screening tool than waist circumference and BMI for adult cardiometabolic risk factors: systematic review and meta-analysis. Obes Rev 2012;13:275-86. 
18. Kahn HS, Bullard KM. Beyond Body Mass Index: Advantages of Abdominal Measurements for Recognizing Cardiometabolic Disorders. Am J Med 2016;129:74-81.

19. Lam BC, Koh GC, Chen C, et al. Comparison of Body Mass Index (BMI), Body Adiposity Index (BAl), Waist Circumference (WC), WaistTo-Hip Ratio (WHR) and Waist-To-Height Ratio (WHtR) as predictors of cardiovascular disease risk factors in an adult population in Singapore. PLoS One 2015;10:e0122985.

20. Ashwell M, Gibson S. Waist-to-height ratio as an indicator of 'early health risk': simpler and more predictive than using a 'matrix' based on BMI and waist circumference. BMJ Open 2016;6:e010159.

21. Savva SC, Lamnisos D, Kafatos AG. Predicting cardiometabolic risk: waist-to-height ratio or BMI. A meta-analysis. Diabetes Metab Syndr Obes 2013;6:403-19.

22. Sardinha LB, Santos DA, Silva AM, et al. A Comparison between BMI, Waist Circumference, and Waist-To-Height Ratio for Identifying Cardio-Metabolic Risk in Children and Adolescents. PLoS One 2016;11:e0149351.

23. Zhao M, Bovet P, Ma C, et al. Performance of different adiposity measures for predicting cardiovascular risk in adolescents. Sci Rep 2017;7:43686

24. Millar SR, Perry IJ, Phillips CM. Assessing cardiometabolic risk in middle-aged adults using body mass index and waist-height ratio: are two indices better than one? A cross-sectional study. Diabetol Metab Syndr 2015;7:73.

25. Huxley R, Mendis S, Zheleznyakov E, et al. Body mass index, waist circumference and waist:hip ratio as predictors of cardiovascular risk--a review of the literature. Eur J Clin Nutr 2010;64:16-22.

26. Carmienke S, Freitag MH, Pischon T, et al. General and abdominal obesity parameters and their combination in relation to mortality: a systematic review and meta-regression analysis. Eur J Clin Nutr 2013;67:573-85.

27. Aeberli I, Gut-Knabenhans I, Kusche-Ammann RS, et al. Waist circumference and waist-to-height ratio percentiles in a nationally representative sample of 6-13 year old children in Switzerland. Swiss Med Wkly 2011;141:w13227.

28. Aeberli I, Gut-Knabenhans M, Kusche-Ammann RS, et al. A composite score combining waist circumference and body mass index more accurately predicts body fat percentage in 6- to 13-yearold children. Eur J Nutr 2013:52:247-53.

29. Freund N, Friedli BC, Junker T, et al. Cardiovascular Risk Assessment and Effects on Behavior in Switzerland The Swiss Heart Foundation HerzCheck $(囚) /$ Cardio-Test $(囚)$. Open Cardiovasc Med J 2015;9:35-9.

30. Chappuis H, Bochud M, Glatz N, et al. Swiss Survey on Salt Intake: Main Results. Lausanne: Federal Office of Public Health, 2011.

31. Marques-Vidal P, Bochud M, Mooser V, et al. Prevalence of obesity and abdominal obesity in the Lausanne population. BMC Public Health 2008;8:330.

32. Bochud M, Beer-Borst S. Anthropometric characteristics and indicators of eating and physical activity behaviors in the Swiss adult population. Results from menuCH 2014-2015. Bern: BAG, 2017.

33. Ogna A, Forni Ogna V, Bochud M, et al. Prevalence of obesity and overweight and associated nutritional factors in a population-based Swiss sample: an opportunity to analyze the impact of three different European cultural roots. Eur J Nutr 2014;53:1281-90.

34. Santi M, Lava SA, Simonetti GD, et al. Clustering of cardiovascular disease risk factors among male youths in Southern Switzerland: preliminary study. Swiss Med Wkly 2016;146:w14338.
35. Carslake D, Jeffreys M, Davey Smith G. Being overweight in early adulthood is associated with increased mortality in middle age. Sci Rep 2016;6:36046.

36. WHO. Waist Circumference and Waist-Hip Ratio. Geneva: WHO, 2008.

37. Wyss T, Marti B, Rossi S, et al. Assembling and Verification of a Fitness Test Battery for the Recruitment of the Swiss Army and Nation-wide Use. Swiss J Sportsmed Sportstraumat 2007;55:126-31.

38. WHO. A global brief on Hypertension. Geneva: WHO, 2013.

39. Ganzeboom H, 2010. A New International Socio-Economic Index (ISEI) of Occupational Status for the International Standard Classification of Occupation 2008 (ISCO 08). Constructed with Data from the ISSP 2002-2007. Annual Conference of International Social Survey Programme. Lisbon

40. Ganzeboom HBG, De Graaf PM, Treiman DJ. A standard international socio-economic index of occupational status. Soc Sci Res 1992;21:1-56.

41. Altman D. Practical statistics for medical research. Boca Raton: Chapman \& Hall/CRC, 1999.

42. Bruggisser M, Burki D, Haeusler M, et al. Multivariable analysis of total cholesterol levels in male Swiss Armed Forces conscripts 2006$2012(\mathrm{~N}=174,872)$. BMC Cardiovasc Disord 2016;16:43.

43. Panczak R, Held L, Moser A, et al. Finding big shots: small-area mapping and spatial modelling of obesity among Swiss male conscripts. BMC Obes 2016;3:10.

44. Malatesta D. Gültigkeit und Relevanz des Body-Mass-Index (BMI) als Massgrösse für Übergewicht und Gesundheitszustand auf individueller und epidemiologischer Ebene. Bern: Gesundheitsförderung Schweiz, 2013.

45. Keys A, Fidanza F, Karvonen MJ, et al. Indices of relative weight and obesity. J Chronic Dis 1972;25:329-43.

46. Swainson MG, Batterham AM, Tsakirides C, et al. Prediction of whole-body fat percentage and visceral adipose tissue mass from five anthropometric variables. PLoS One 2017:12:e0177175.

47. Chiolero A, Paradis G, Maximova K, et al. No use for waist-for-height ratio in addition to body mass index to identify children with elevated blood pressure. Blood Press 2013;22:17-20.

48. Clair C, Chiolero A, Faeh D, et al. Dose-dependent positive association between cigarette smoking, abdominal obesity and body fat: cross-sectional data from a population-based survey. BMC Public Health 2011;11:23.

49. Marques-Vidal P, Bochud M, Mooser V, et al. Obesity markers and estimated 10-year fatal cardiovascular risk in Switzerland. Nutr Metab Cardiovasc Dis 2009;19:462-8.

50. Wallner A, Hirz A, Schober E, et al. Evolution of cardiovascular risk factors among 18-year-old males in Austria between 1986 and 2005. Wien Klin Wochenschr 2010;122:152-8.

51. Nádas J, Putz Z, Kolev G, et al. Intraobserver and interobserver variability of measuring waist circumference. Med Sci Monit 2008:14:CR15-18.

52. Sebo P, Haller D, Pechère-Bertschi A, et al. Accuracy of doctors' anthropometric measurements in general practice. Swiss Med Wkly 2015;145:w14115.

53. Higgins PB, Comuzzie AG. Measures of Waist Circumference. Preedy VR, ed. Handbook of Anthropometry: Physical Measures of Human Form in Health and Disease. Berlin, Germany: Springer, 2012:881-91.

54. Luz RH, Barbosa AR, d'Orsi E. Waist circumference, body mass index and waist-height ratio: Are two indices better than one for identifying hypertension risk in older adults? Prev Med 2016;93:76-81. 